\title{
The effect of different alkaline catalysts on the formation of silica aerogels prepared by the sol-gel approach
}

\author{
Yujing WANG ${ }^{1}$, Junnan HAN ${ }^{1,}$, Jiexiu ZHAI ${ }^{1}$ and Daling YANG ${ }^{1}$ \\ ${ }^{1}$ Department of Civil Engineering, Dalian University of Technology, Dalian 116024, P. R. China
}

Due to the unique three-dimensional spatial network structure formed by $\mathrm{SiO}_{2}$ nanoparticles and high surface aera, silica aerogel is a high-profile material in many fields. In the present study, by adopting the industrial water glass as the silicon source, silica aerogels had been prepared via the sol-gel approach followed by the ambient pressure drying process. The effects of different alkaline catalysts in terms of $\mathrm{LiOH}, \mathrm{NaOH}, \mathrm{KOH}$, and $\mathrm{NH}_{3} \cdot \mathrm{H}_{2} \mathrm{O}$ on the formation of silica aerogels were investigated. It was found that with the introduction of alkaline catalysts, the gel aging time dramatically decreased from 5840 to $31 \mathrm{~min}$ during the sol-gel process. Compared with the silica aerogels prepared without any catalyst, accordingly, the thermal stability was significantly improved, and the decomposition temperature increased from 375 to above $410{ }^{\circ} \mathrm{C}$. Furthermore, the silica aerogels prepared with alkaline catalysts were featured in a distinctive double-pore structure. It was shown that the alkali metal ions might have an alkaline dissolution effect in the weakly acidic environment during the sol-gel process. Moreover, this effect might increase the contact area between different $\mathrm{SiO}_{2}$ nanoparticles, which was benefit to maintain the three-dimensional spatial network structure and resulted in the silica aerogels with higher thermal stability. The alkaline dissolution effect had an influence on the pore size distribution of silica aerogels with a double pore structure at 1.7 and $32 \mathrm{~nm}$ as well as retained large pore volume at about $2.60-3.35 \mathrm{~cm}^{3} / \mathrm{g}$. (02020 The Ceramic Society of Japan. All rights reserved.

Key-words : Alkaline catalysts, Silica aerogels, Sol-gel, Alkaline dissolution effect

[Received December 18, 2019; Accepted March 30, 2020]

\section{Introduction}

In 1931, Kistler first created the aerogels in the laboratory. ${ }^{1)}$ Since then, the research of aerogels has never stopped. Until now, the synthesis and application of aerogels are still hotspots in the field of porous materials. Silica aerogels have some extraordinary properties compared with other kinds of aerogels, due to their unique three-dimensional spatial network structure formed by $\mathrm{SiO}_{2}$ nanoparticles, which contains over $95 \%$ air and less than $5 \%$ skeleton of the whole volume. ${ }^{2)}$ Some of the specific parameters can be summarized as follows: specific surface area $\left(600-800 \mathrm{~m}^{2} / \mathrm{g}\right)$, thermal conductivity $[0.017-$ $0.021 \mathrm{~W} /(\mathrm{m} \mathrm{K})]$, pore size $(5-100 \mathrm{~nm})$ and total porosity $\left(90\right.$ vol \%). $\left.{ }^{3}\right)$ Silica aerogels with such excellent properties mentioned above can be used in many fields, such as aerospace, ${ }^{4)}$ catalysts for gas-phase reactions, ${ }^{5)}$ drug carrier systems, ${ }^{6)}$ new material battery, ${ }^{7)}$ heat and sound insulation, ${ }^{8)}$ etc.

At present, the primary preparation method of silica aerogels is the sol-gel approach. During the sol-gel process, it may be affected by various factors, such as the type of silicon source, ${ }^{9)-12)}$ the ratio of $\mathrm{Na}_{2} \mathrm{SiO}_{3} / \mathrm{H}_{2} \mathrm{O},{ }^{13)} \mathrm{pH}$ of

Corresponding author: J. Han; E-mail: junnanhan@dlut. edu.cn silica sol-solution, ${ }^{14)}$ aging process, ${ }^{15), 16)}$ type and concentration of catalysts, ${ }^{17), 18)}$ drying method ${ }^{19), 20)}$ etc. All these factors will have a profound influence on the structure and performance of the final silica aerogels products. For example, Wagh et al. ${ }^{21)}$ researched the influence of different silicon sources: tetraethoxysilane (TMOS), tetraethylorthosilicate (TEOS) and polyethoxydisiloxane (PEDS) on the preparation of silica aerogels, which concluded that the silica aerogels prepared with TMOS had more uniform pore distribution and lower surface area compared with TEOS and PEDS. Rao et al. ${ }^{13)}$ systematically investigated the influence of $\mathrm{Na}_{2} \mathrm{SiO}_{3} / \mathrm{H}_{2} \mathrm{O}$ on the properties of silica aerogels prepared with sodium silicate, indicating that the bulk density, percentage of volume shrinkage and optical transmission all decreased with an increase of $\mathrm{Na}_{2} \mathrm{SiO}_{3} /$ $\mathrm{H}_{2} \mathrm{O}$ in the initial ratio. Rao et al. ${ }^{18)}$ explored the influence of catalyst concentration on the properties of silica aerogels, which revealed that silica aerogels exhibited conspicuous (distinctive) single crystal property and transparency when the concentration of $\mathrm{HCl}$ is $0.01 \mathrm{~N}$. Zhao et al. ${ }^{19)}$ demonstrated that the silica aerogels prepared via the freeze-drying method showed larger specific surface areas compared with the ambient drying process. Torres et al. ${ }^{20)}$ had synthesized the fiber-reinforced tetraethylorthosilicate/vinyltrimethoxysilane (TEOS/VTMS) derived silica aerogels by adopting ambient pressure drying 
and supercritical conditions drying method, respectively. Also, the comparative analysis indicated that ambient pressure drying was an alternative approach to obtain highly porous aerogels.

Generally, most of the previous researches were used $\mathrm{NH}_{3} \cdot \mathrm{H}_{2} \mathrm{O}$ or $\mathrm{NaOH}$ as the alkaline catalysts in the transformation process from silica sol to silica gel. It was found that the ions of $\mathrm{Na}^{+}$from the residual silicon precursor or introduced by $\mathrm{NaOH}$ added in the silica sol would have a significant influence on the performance of the silica aerogels. ${ }^{22), 23)}$ Unfortunately, the influence of different alkaline catalysts with different alkali metal ions on the silica aerogels fabrication usually seems to be neglected. Therefore, it is significant to explore the effect of different alkali metal ions on the formation of silica aerogels by the solgel approach.

In this study, hydrophobic and light silica aerogels were prepared by using low-cost industrial sodium silicate as silicon source via ambient pressure drying process. In the sol-gel progress, alkaline catalysts with different alkali metal ions of $\mathrm{Li}^{+}, \mathrm{Na}^{+}, \mathrm{K}^{+}$were introduced to investigate the alkaline effect. For preciseness, the comparative experiments with $\mathrm{NH}_{3} \cdot \mathrm{H}_{2} \mathrm{O}$ as the alkaline catalyst and blank test without any alkaline catalyst were also set in this paper.

\section{Experimental}

\subsection{Raw materials}

The raw chemical materials used were listed in Table $\mathbf{1 .}$

\subsection{Preparation methods}

\subsubsection{Preparation of silica alcogels}

Water glass was hydrolyzed in deionized water at the molar ratio of 1:4 for $30 \mathrm{~min}$. After that, the hydrolyzed solution was ion-exchanged with strong acid styrene cation exchange resin, which could replace $\mathrm{Na}^{+}$and other foreign ions with $\mathrm{H}^{+}$in the solution. ${ }^{24)} \mathrm{The}_{\mathrm{pH}}$ of the obtained silicate solution was 2.00. And then the silica sol was divided into five equal parts, which were numbered as $\mathrm{T}-0, \mathrm{~T}-1, \mathrm{~T}-2, \mathrm{~T}-3$, and T-4. The $\mathrm{pH}_{2}$ of silica sols T-1, T-2, $\mathrm{T}-3$, and $\mathrm{T}-4$ were modified to $5.15-5.25$ by $\mathrm{LiOH}, \mathrm{NaOH}$, $\mathrm{KOH}$ and $\mathrm{NH}_{3} \cdot \mathrm{H}_{2} \mathrm{O}$, respectively. The molarity of four alkaline catalysts used was $1.0 \mathrm{~mol} \cdot \mathrm{L}^{-1}$. Silica sols $\mathrm{T}-0$, $\mathrm{T}-1, \mathrm{~T}-2, \mathrm{~T}-3$, and T-4 were placed until gelation occurred.
For further aging, deionized water was replenished, and then the silica gels were sealed and placed into a water bath at $50^{\circ} \mathrm{C}$ for $24 \mathrm{~h}$. In addition, different volume percentage concentrations of ethanol $(30,60,100$ vol-\%) were doped successively by a separate-step impregnation method to exchange the water in the pores of silica gels with a water bath at $50^{\circ} \mathrm{C}$ for $24 \mathrm{~h}$.

\subsubsection{Preparation of silica aerogels}

Silica aerogels were prepared with hydrophobic modification followed by ambient pressure drying process. The prepared silica alcogels were placed in n-hexane (nonpolar solvent $)^{25)}$ and sealed in a water bath at $50^{\circ} \mathrm{C}$ for $24 \mathrm{~h}$ to replace some water and ethanol in the network structure of silica alcogels. Then, Trimethylchlorosilane (TMCS)/nhexane modification solution was added, and hydrophobic modification was performed at $50{ }^{\circ} \mathrm{C}$ for $24 \mathrm{~h}$, where the initial mass of TMCS: n-Hexane: silica gel was 1.5:2:1. After that, the modified silica gels were placed in an nHexane solution with two times initial mass in the temperature at $50^{\circ} \mathrm{C}$ for $24 \mathrm{~h}$ to wash out the rest modified solution and secondary by-products, such as $\mathrm{HCl}$, hydrolysis or alcoholysis products of TMCS. Furthermore, the silica gel was placed in a constant temperature drying box at $50,80,120$, and $180^{\circ} \mathrm{C}$ for $2 \mathrm{~h}$, respectively. Finally, the light and hydrophobic silica aerogels were prepared. The experimental flowchart was displayed in Fig. 1.

\subsection{Characterization}

The bulk density $(\rho)$ of the produced silica aerogels was measured by using the formula $\rho=m / V$ where $m$ respects mass of the sample weighed by the analytical balance and $V$ is volume measured by the drainage method. The EQUINOX55 Fourier transform spectrometer from Bruker, Germany was used to determine the functional groups on the surface of silica aerogels, of which the scanning range was $4000-400 \mathrm{~cm}^{-1}$. The thermal stability test was carried out by the Swiss Mettler Toledo DGA/DSC 1 synchronous thermal analyzer. The heating rate was $10^{\circ} \mathrm{C} / \mathrm{min}$, and the temperature range was $50-950^{\circ} \mathrm{C}$. The microstructure and pore structure of silica aerogels were observed by NOVA NanoSEM 450 ultra-high resolution field emission scanning electron microscope. The BET method was used to measure the specific surface area of the silica aerogels by Autosorb-1mp BET automatic adsorption

Table 1. Raw chemical materials used in the experiment

\begin{tabular}{lccl}
\hline Chemical reagent name & Molecular formula & Purity & \multicolumn{1}{c}{ Manufacturer } \\
\hline sodium silicate & - & $\mathrm{Na}_{2} \mathrm{O}: \mathrm{SiO}_{2}$ molar ratio $=1: 3.1$, & Dalian Qing'an Chemical Co., Ltd., China \\
solid content $=30 \%$ & Sinopsin Chemical Reagent Co., Ltd., China \\
strong acid styrene & - & - & Tianjin Guangfu Technology Development Co., Ltd., China \\
cation exchange resin & & AR & Tianjin Kemio Chemical Reagent Co., Ltd., China \\
Ethanol(EtOH) & $\mathrm{CH}_{3} \mathrm{CH}_{2} \mathrm{OH}$ & AR & Tianjin Kemio Chemical Reagent Co., Ltd., China \\
n-Hexane & $\mathrm{CH}_{3}\left(\mathrm{CH}_{2}\right)_{2} \mathrm{CH}_{3}$ & AR & Jilin Xinyaqiang Industrial Co., Ltd., China \\
Ammonia water & $\mathrm{NH}_{3} \cdot \mathrm{H}_{2} \mathrm{O}$ & AR & Shandong Xiya Chemical Industry Co., Ltd., China \\
TMCS & $\left(\mathrm{CH}_{3}\right)_{3} \mathrm{SiCl}$ & AR & Sinopsin Chemical Reagent Co., Ltd., China \\
Lithium hydroxide & $\mathrm{LiOH}$ & AR & Tianjin Damao Chemical Reagent Factor., China \\
Sodium hydroxide & $\mathrm{NaOH}$ & AR & fabricated in laboratory with a resistivity of 18 M 2 cm \\
Potassium hydroxide & $\mathrm{KOH}$ & - &
\end{tabular}




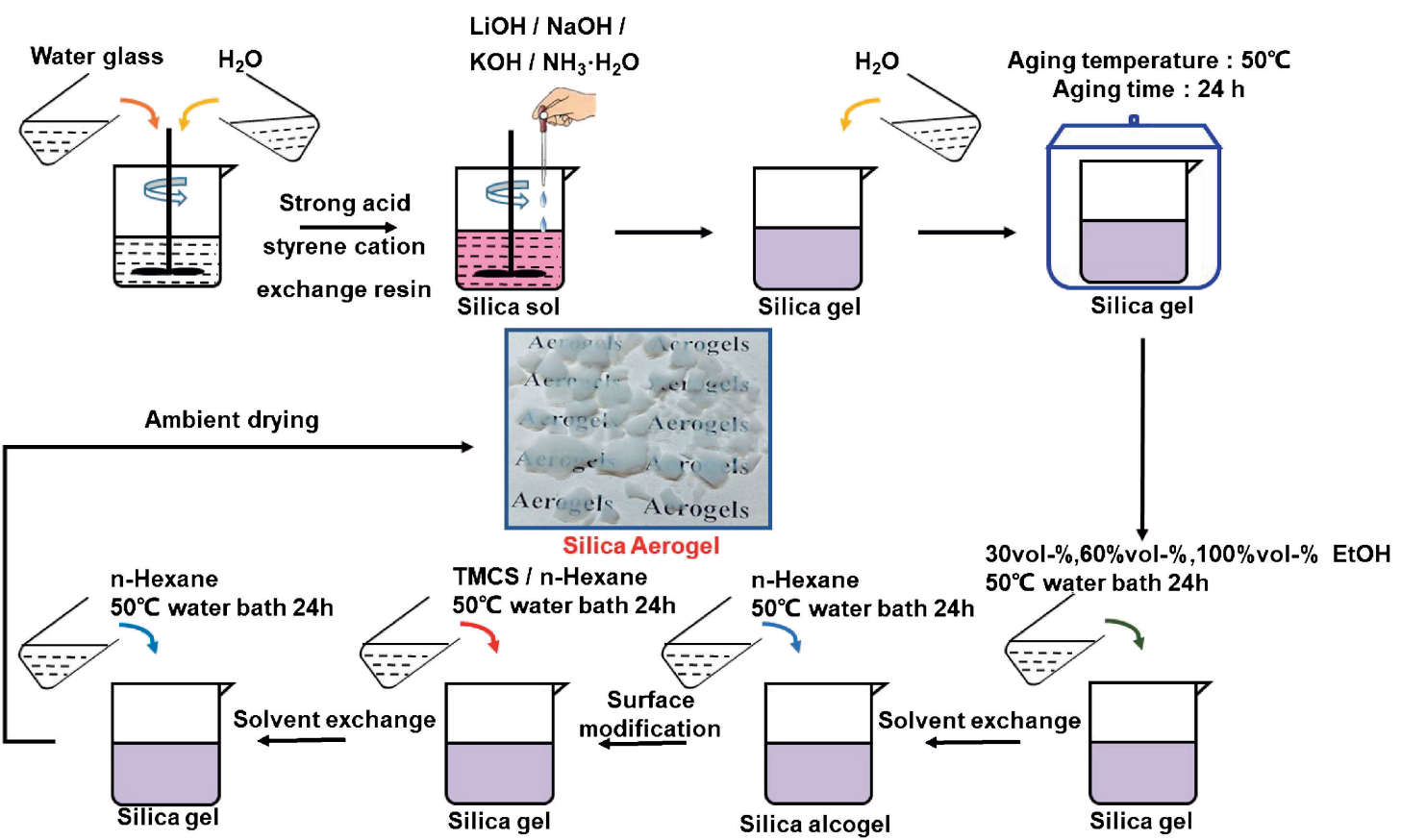

Fig. 1. Experimental flowchart of the preparation of silica aerogels.

instrument of the Quantachrome company. The pore size distribution and pore volume were calculated from the desorption branch of the isotherm by the Barrett-JoynerHalender model, despite the known limitations of nitrogen sorption analysis in determining pore size and pore volume. ${ }^{26)}$ Accordingly the adsorption mass was $\mathrm{N}_{2}$, degassing temperature was $200^{\circ} \mathrm{C}$, and degassing time was $3 \mathrm{~h}$.

\section{Results and discussion}

\subsection{Chemical structure}

Fourier Transform Infra-Red Spectroscopy (FT-IR) of silica aerogels prepared with different alkaline catalysts are shown in Fig. 2. There was a relatively broad absorption peak centered at $3435 \mathrm{~cm}^{-1}$, which was the infrared absorption peak of asymmetric stretching vibration of $-\mathrm{OH}$ bond. ${ }^{27)}$ The absorption peak centered at $1631 \mathrm{~cm}^{-1}$ was associated with the bending vibration of the $\mathrm{H}-\mathrm{O}-\mathrm{H}$ bond, which came from absorbed water. ${ }^{28), 29)}$ The peak centered at $947 \mathrm{~cm}^{-1}$ was attributed to the stretching vibration peak of the $\mathrm{Si}-\mathrm{OH}$ group. ${ }^{30)}$ The anti-symmetric stretching vibration absorption peak, the symmetric stretching vibration peak, and the bending vibration peak of Si-O-Si bond were ascribed at 1097,802 and $470 \mathrm{~cm}^{-1}$, respectively. ${ }^{31)}$ The peaks centered at 2964,2918 , and $2850 \mathrm{~cm}^{-1}$ were generated by stretching vibration of $\mathrm{C}-\mathrm{H}$ bond; the peaks centered at $1256,846 \mathrm{~cm}^{-1}$ were vibration absorption peaks of $\mathrm{Si}-\mathrm{C}$ bond; ${ }^{32)}$ and the peak centered at $758 \mathrm{~cm}^{-1}$ was the $\mathrm{C}-\mathrm{H}$ plane sway flexural vibration of the methyl group. The existence of these absorption peaks and the separately listed infrared spectra of silica aerogels prepared by $\mathrm{LiOH}$ as catalyst confirmed that all prepared aerogels contain $-\mathrm{CH}_{3}$ groups. ${ }^{33), 34)}$ As presented in Fig. 2, however, the stretching vibration peak of $\mathrm{Si}-\mathrm{OH}$ at $947 \mathrm{~cm}^{-1}$ still existed with a low absorption intensity. The presence of a small amount of $\mathrm{Si}-\mathrm{OH}$ group resulted that the silica aerogels broke up into smaller pieces and tended to crack when dried via ambient pressure drying process. In the periodic table of elements, with the increase of the atomic number of the metal (main group) of the same group, the alkalinity of the hydrate (hydroxide) corresponding to the highest oxide of the metal is stronger. What's more, the alkaline of $\mathrm{NH}_{3} \cdot \mathrm{H}_{2} \mathrm{O}$ is weak because it disassociates little $\mathrm{OH}^{-}$ions in water. So that the order of the alkaline catalysts used in the experiment is $\mathrm{LiOH}>\mathrm{NaOH}>\mathrm{KOH}>$ $\mathrm{NH}_{3} \cdot \mathrm{H}_{2} \mathrm{O}$. From the infrared spectra of silica aerogels prepared by four different alkaline catalysts, it's clear that, as the alkalinity decreased, the strength of methyl characteristic absorption peak at 2964, 2918, $2850 \mathrm{~cm}^{-1}$ increased. The samples pre-treated by using $\mathrm{NH}_{3} \cdot \mathrm{H}_{2} \mathrm{O}$ as catalyst exhibited an increase at $846 \mathrm{~cm}^{-1}$, indicating $\mathrm{Si}-\mathrm{C}$ vibration enhanced. A new peak at $1408 \mathrm{~cm}^{-1}$ belongs to methyl's C-H shear-type bending vibration, which was caused by hydrophobic modification of TMCS.

\subsection{Basic physical properties}

Several detailed parameters of the fundamental physical properties of silica aerogels were listed in Table 2. In order to shorten the aging time during the sol-gel process, the $\mathrm{pH}$ of silica sol obtained by the ion-exchange method was adjusted from 2.00 to about 5.20. As indicated in Table 2, with the introduction of alkaline catalysts, the gel aging time was dramatically decreased from 5840 to 31 min during the sol-gel process. Moreover, the addition of alkaline catalysts didn't change the properties of low bulk density and high porosity of silica aerogels prepared.

\subsection{Thermal properties}

Thermal Gravity (TG) analysis and Differential Thermal 


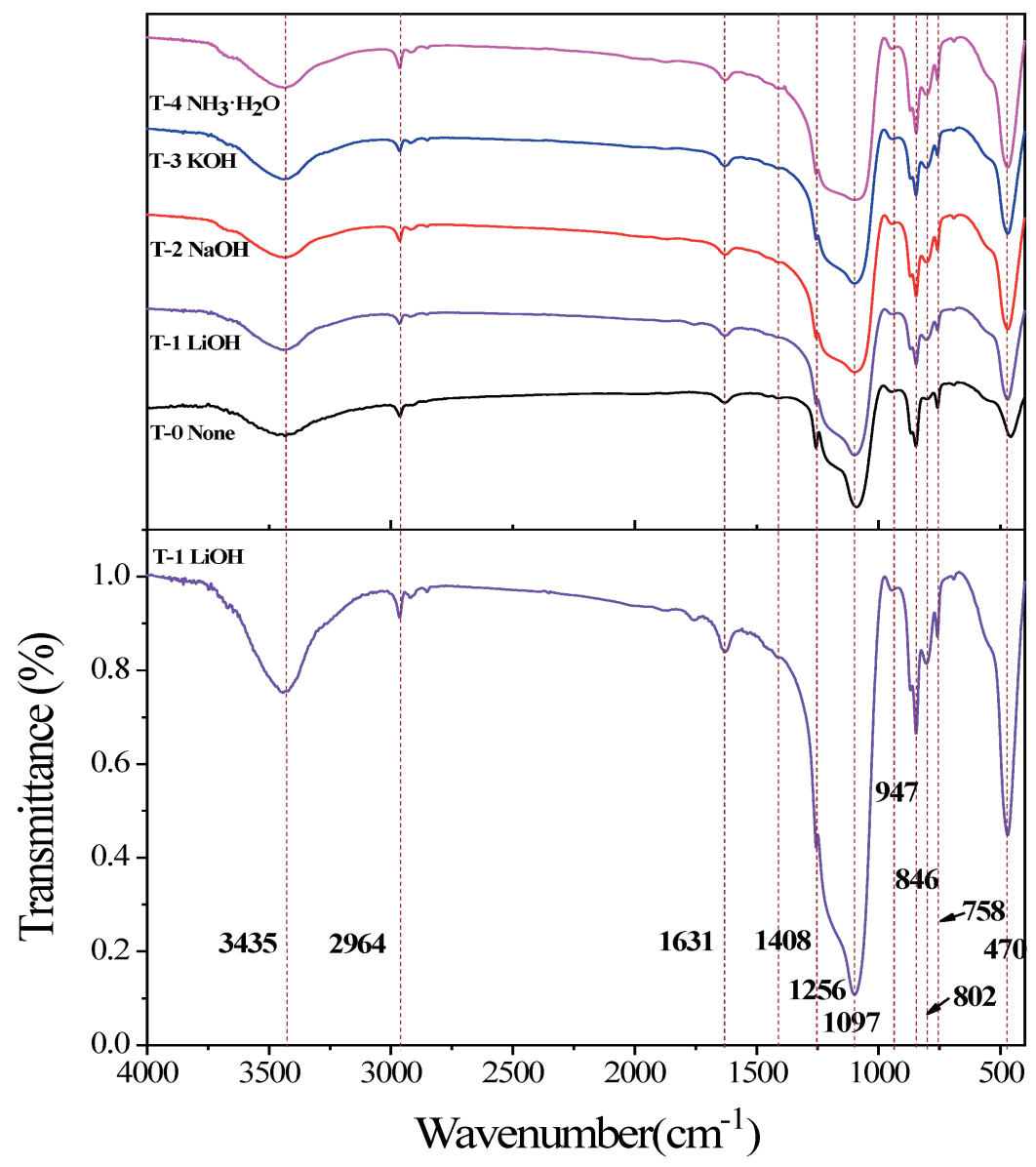

Fig. 2. FT-IR of silica aerogels prepared with different alkaline catalysts (In order to show the characteristic peaks more clearly, the FT-IR of silica aerogels using LiOH as catalyst was listed separately.).

Table 2. Basic physical properties of silica aerogels prepared with different alkaline catalysts

\begin{tabular}{cccccc}
\hline \multirow{2}{*}{ Samples } & \multirow{2}{*}{$\mathrm{pH}_{1}$} & $\mathrm{pH}_{2}$ & Gel transition time & Bulk density & Porosity \\
\cline { 4 - 6 } & & & $\min$ & $\mathrm{g} / \mathrm{cm}^{3}$ & $\%$ \\
\hline $\mathrm{T}-0$ & 2.00 & 2.00 & 5840 & 0.111 & 94.9 \\
$\mathrm{~T}-1$ & 2.00 & 5.17 & 31 & 0.114 & 94.8 \\
$\mathrm{~T}-2$ & 2.00 & 5.20 & 46 & 0.129 & 94.1 \\
$\mathrm{~T}-3$ & 2.00 & 5.24 & 37 & 0.099 & 95.5 \\
$\mathrm{~T}-4$ & 2.00 & 5.17 & 36 & 0.107 & 95.1 \\
\hline
\end{tabular}

*T-0: None; T-1: LiOH; T-2: $\mathrm{NaOH}$; T-3: $\mathrm{KOH}$; $\mathrm{T}-4$ : $\mathrm{NH}_{3} \cdot \mathrm{H}_{2} \mathrm{O}$; Bulk density $\rho=(m / v)$, where $m$ is the mass of silica aerogels and $v$ is the volume measured by drainage method; Porosity $\mathrm{P}(\%)=100 \% \times[1-$ $\left.\left(\rho / \rho_{\mathrm{SiO}_{2}}\right)\right]\left(\rho_{\mathrm{SiO}_{2}}=2.19 \mathrm{~g} / \mathrm{cm}^{3}\right)$.

Gravity (DTG) analysis curves of silica aerogels prepared with different alkaline catalysts were shown as Fig. 3. It was revealed that with the introduction of alkaline catalysts, the thermal stability of silica aerogels had been improved, which was embodied in that the total mass loss had been reduced sharply from 10.42 to $7.24 \%$. What's more, with the addition of alkaline catalysts containing alkali metal ions, the total mass loss of silica aerogels was even lower. For the blank group T-0 [Fig. 3(b)], the weight loss peak at $245^{\circ} \mathrm{C}$ was caused by the residual organic matter in the aerogel combustion. ${ }^{35)}-\mathrm{OH}$ bonds at the open hole on the surface of the silicon were replaced with the
$-\mathrm{O}-\mathrm{Si}-\left(\mathrm{CH}_{3}\right)_{3}$ groups as a result of hydrophobic modification. As shown in Figs. 3(b)-3(f), the rapid weight loss peak of blank sample $\mathrm{T}-0$ at $376^{\circ} \mathrm{C}$ indicated that $-\mathrm{CH}_{3}$ began to oxidize. ${ }^{13)}$ With the using of alkaline catalysts, the decomposition temperature of silica aerogels rose from 376 to $400-410{ }^{\circ} \mathrm{C}$ [Figs. 3(b)-3(f)].

According to the previous research, ${ }^{28)}$ the hydrophobicity of silica aerogels prepared by hydrophobic modification could keep its performance up to $500^{\circ} \mathrm{C}$. Once the temperature reached $500^{\circ} \mathrm{C}$, the $-\mathrm{CH}_{3}$ groups introduced by surface modification were wholly oxidized to $-\mathrm{OH}$ bond. When the temperature continued to rise, the adjacent $-\mathrm{OH}$ bonds would undergo condensation and dehydration, thus the structure of the whole silica aerogels got to be densified. As illustrated in Table 2, the total mass loss of silica aerogels T-4 prepared by weak alkaline catalyst $\left(\mathrm{NH}_{3} \cdot \mathrm{H}_{2} \mathrm{O}\right)$ is $7.24 \%$, which was higher than that of silica aerogels T-1, T-2, and T-3 prepared by strong alkaline catalysts containing alkali metal ions $(\mathrm{LiOH}, \mathrm{NaOH}$, $\mathrm{KOH})$.

\subsection{Morphology of silica aerogels}

Scanning Electron Microscope (SEM) photos silica aerogels prepared by different alkaline catalysts were displayed as Fig. 4. It can be seen that the three-dimensional spatial network constitutes the main structure of silica 

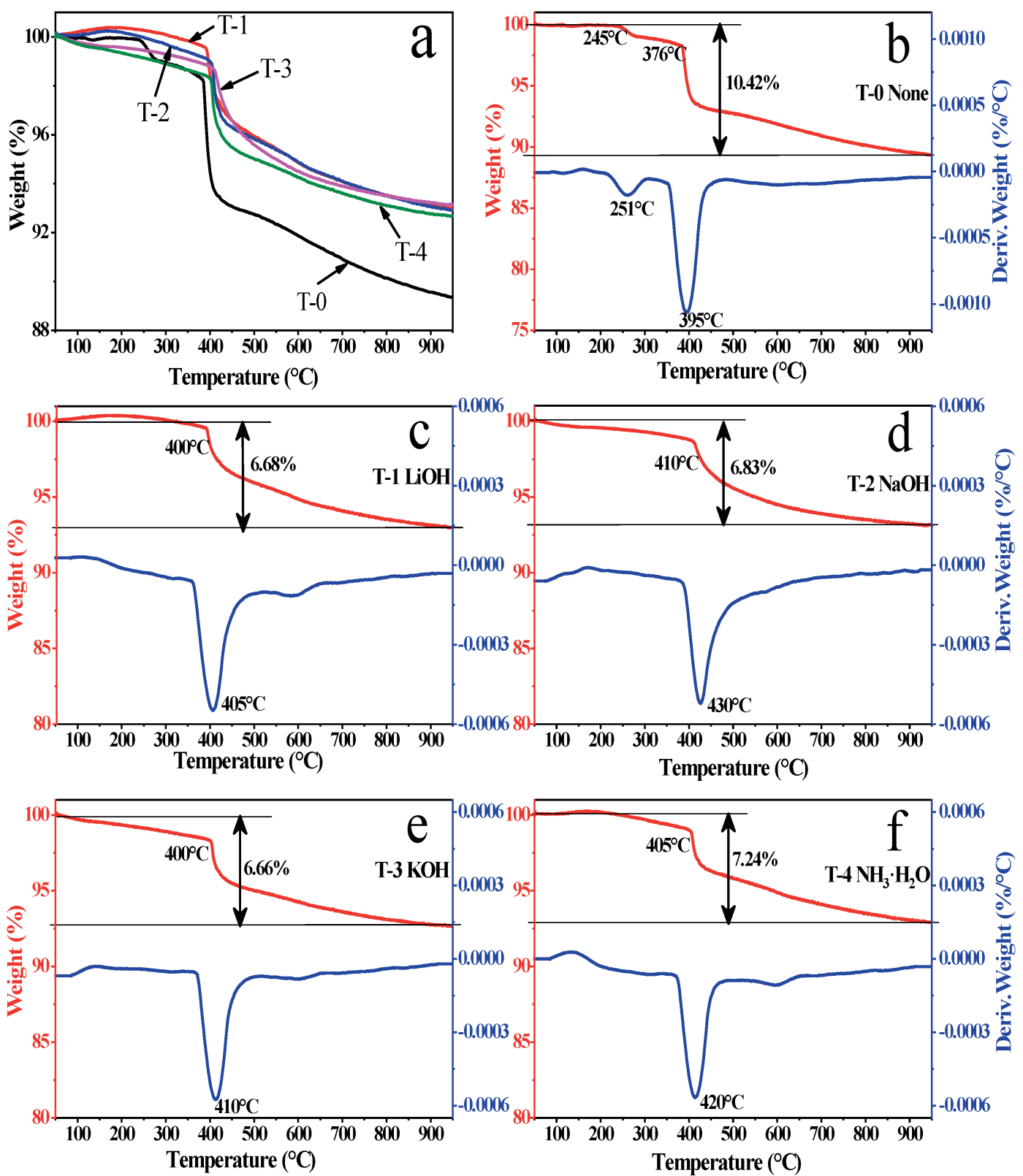

Fig. 3. TG and DTG curves of silica aerogels prepared with different alkaline catalysts: a: TG curves; $b$ (T-0): None; c (T-1): LiOH; d (T-2): $\mathrm{NaOH}$; e (T-3): $\mathrm{KOH}$; (T-4): $\mathrm{NH}_{3} \cdot \mathrm{H}_{2} \mathrm{O}$.

aerogel, which leads to the weak direct connection between particles and particles. This is also the reason why silica aerogels are fragile at the macro level. ${ }^{36}$ ) What's more, regardless of the catalysts adopted, silica aerogels with a three-dimensional spatial network structure formed by $\mathrm{SiO}_{2}$ nanoparticles could be obtained.

The skeletons of the silica aerogels T-4 [Figs. 4(i) and 4(j)] catalyzed with $\mathrm{NH}_{3} \cdot \mathrm{H}_{2} \mathrm{O}$ were mostly $\mathrm{SiO}_{2}$ nanoparticles, while those of the silica aerogels T-1 [Figs. 4(c) and 4(d)], T-2 [Figs. 4(e) and 4(f)], and T-3 [Figs. 4(g) and $4(\mathrm{~h})$ ] catalyzed by $\mathrm{LiOH}, \mathrm{NaOH}$ and $\mathrm{KOH}$ were mainly connected together in a lamellar structure. This structure could increase the connection area between $\mathrm{SiO}_{2}$ clusters. The presence of $\mathrm{Li}^{+}, \mathrm{Na}^{+}$, and $\mathrm{K}^{+}$alkali metal ions might produce an alkaline dissolution effect. Under this effect, the homogenization of $\mathrm{SiO}_{2}$ nanoclusters was facilitated, and the connection area between clusters was increased. At the same time, the three-dimensional spatial network structure of silica aerogels was still maintained.

Transmission Electron Microscope (TEM) images at different magnifications of silica aerogels prepared with different alkaline catalysts were shown as Fig. 5. The skeletons of the silica aerogels T-1 [Figs. 5(a)-5(c)], T-2 [Figs. 5(d)-5(f)], T-3 [Figs. 5(g)-5(i)] and T-4 [Figs. 5(j)5(l)] prepared by using $\mathrm{LiOH}, \mathrm{NaOH}, \mathrm{KOH}$ and $\mathrm{NH}_{3} \cdot \mathrm{H}_{2} \mathrm{O}$ as catalyst showed highly cross-linked sponge-like network structure and the skeleton particles were relatively uniform and transparent. It was noteworthy that with the introduction of alkaline catalysts, the skeleton particles of silica aerogels got to be more uniform. 

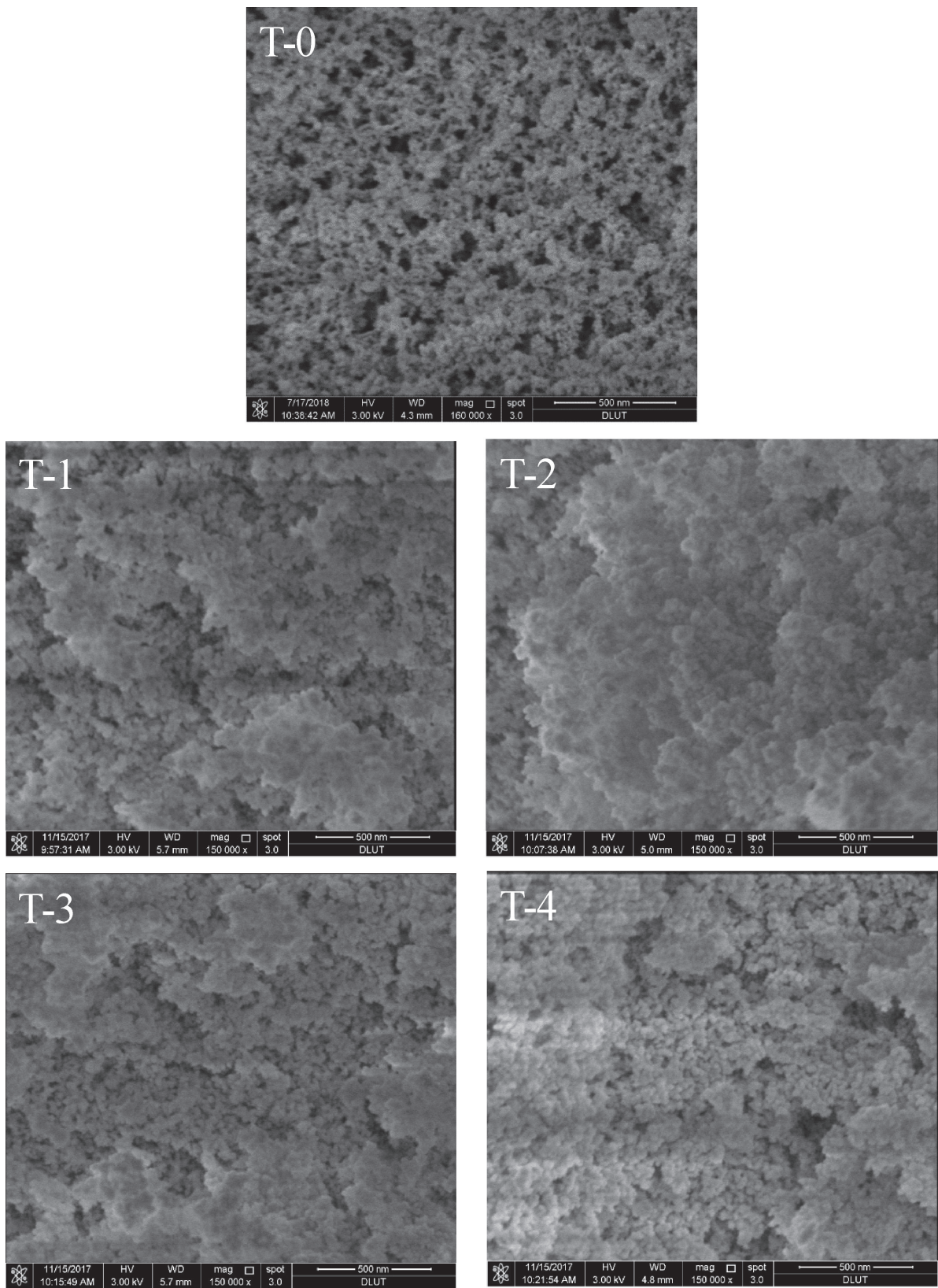

Fig. 4. SEM images at different magnifications of the samples: T-0: None; T-1: LiOH; T-2: NaOH; T-3: KOH; T-4: $\mathrm{NH}_{3} \cdot \mathrm{H}_{2} \mathrm{O}$.

\subsection{Pore structure}

The Nitrogen adsorption-desorption isotherms and the pore size distributions of silica aerogels prepared with different alkaline catalysts were shown as Figs. 6 and 7. In order to facilitate the study of pore size distribution and structure of porous materials, the international union of pure and applied chemistry (IUPAC) ${ }^{37)}$ classified adsorption-desorption isotherms and hysteresis loops of porous materials. According to that, the adsorptiondesorption isotherms of silica aerogels prepared by four different alkaline catalysts belonged to IV type, manifesting that the prepared silica aerogels were a kind of traditional mesoporous materials. Additionally, the H1 hysteresis loop at the medium high-pressure stage was caused by an apparent capillary condensation phenomenon, which indicated that the silica aerogels prepared had a homogeneous mesoporous structure. The alkaline dissolution effect not only increased the contact area in $\mathrm{SiO}_{2}$ nanoclusters but also maintained a porous three-dimensional network structure of silica aerogels. It had also been proved by the pore volume size of silica aerogels T-1, T-2, and T-3 as listed in Table 3. With the introduction of alkaline catalysts, the specific surface area of silica aerogels had changed from 770 to about $400 \mathrm{~m}^{2} / \mathrm{g}$.

According to the pore size distribution of silica aerogels prepared with different alkaline catalysts, silica aerogels T- 0 was to be a heterogeneous-pore material with a pore diameter of $2-30 \mathrm{~nm}$. However, the pore size of silica aerogels $\mathrm{T}-1, \mathrm{~T}-2, \mathrm{~T}-3$, and T-4 were distributed between $1-100 \mathrm{~nm}$. What's more, the silica aerogels prepared with 

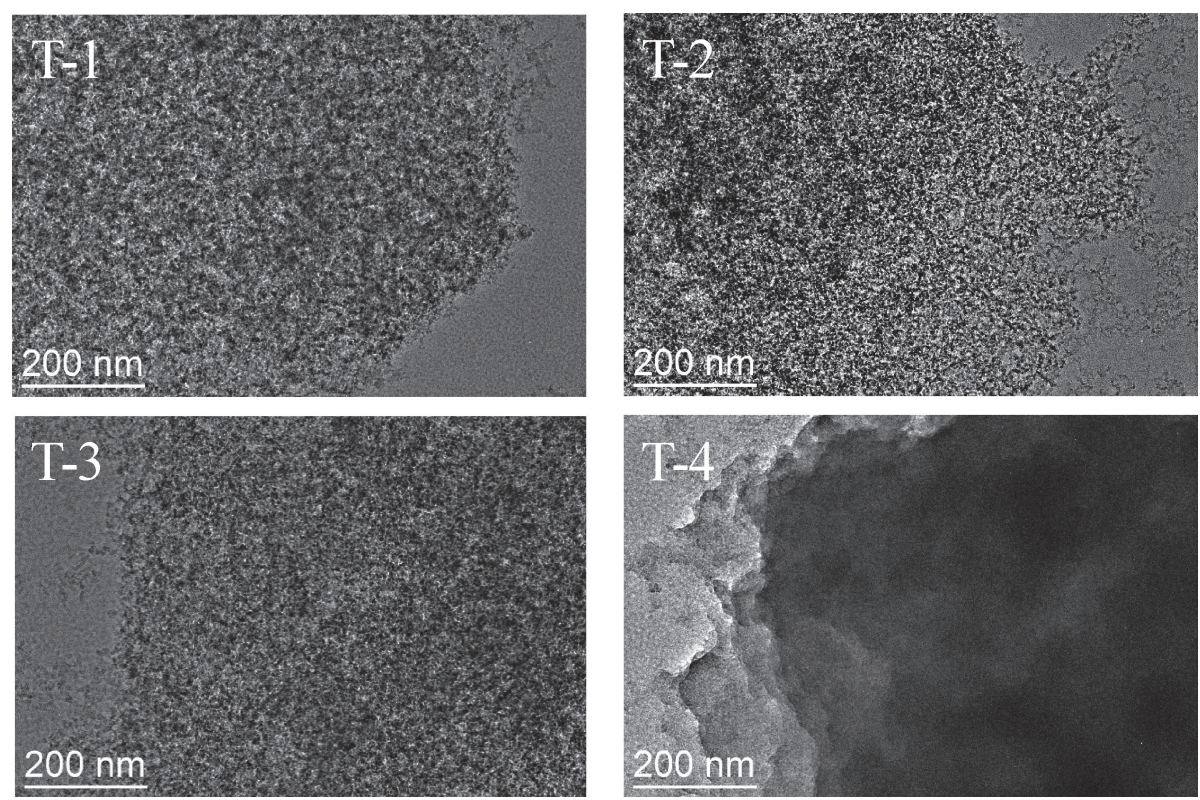

Fig. 5. TEM images at different magnifications of the samples: T-1: LiOH; T-2: NaOH; T-3: KOH; T-4: $\mathrm{NH}_{3} \cdot \mathrm{H}_{2} \mathrm{O}$.

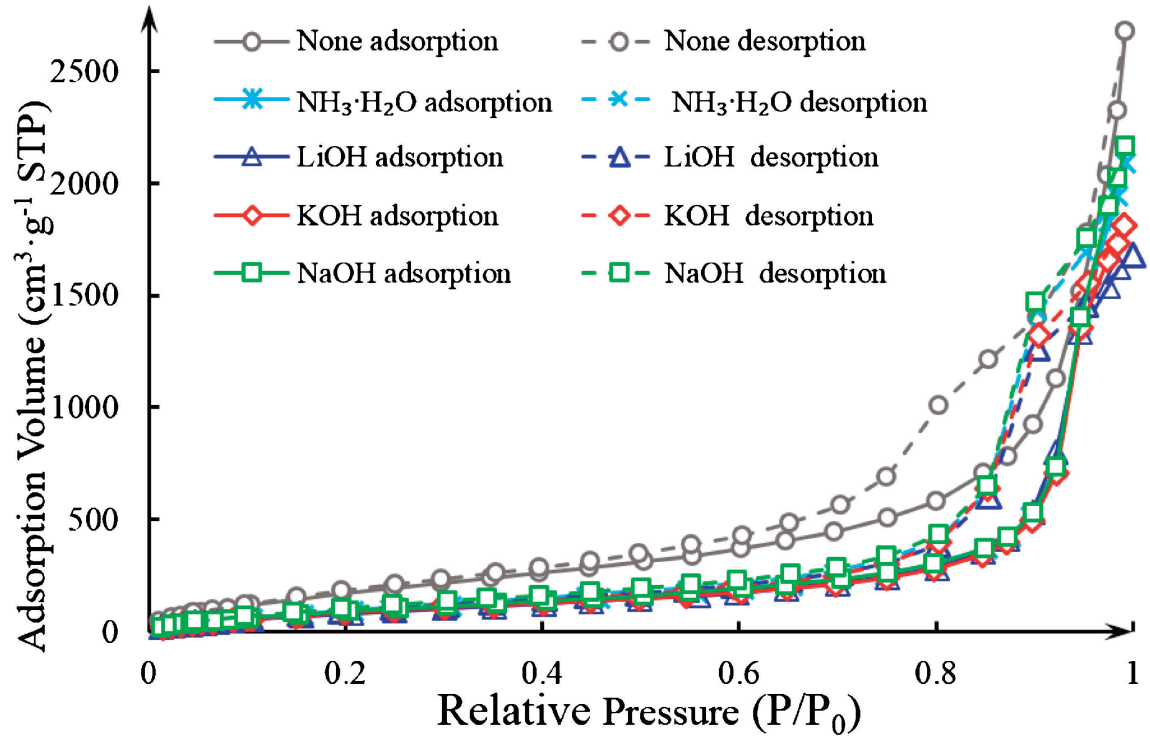

Fig. 6. Nitrogen adsorption-desorption isotherms of silica aerogels prepared with different alkaline catalysts.

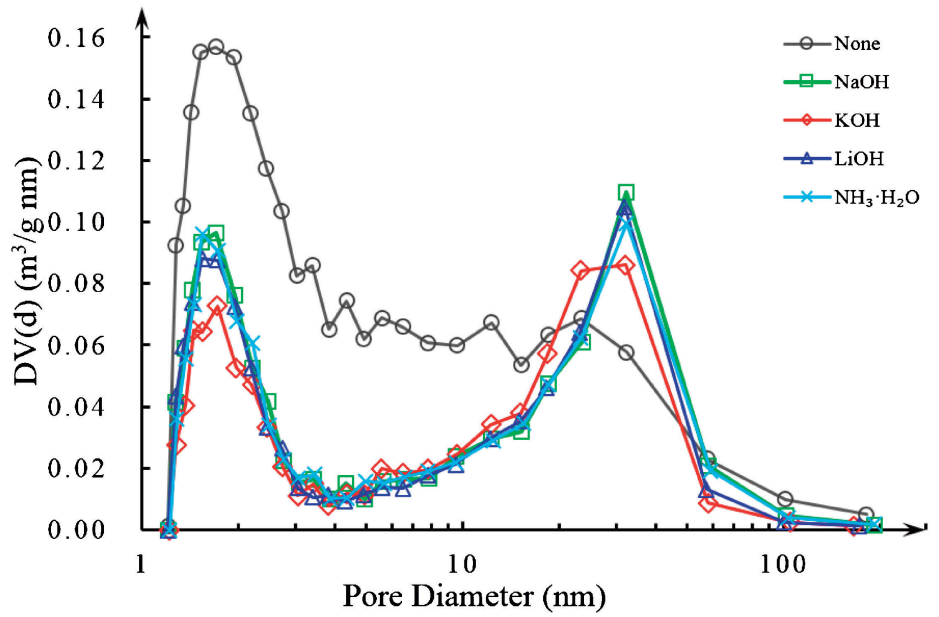

Fig. 7. Pore size distributions of silica aerogels prepared with different alkaline catalysts. 


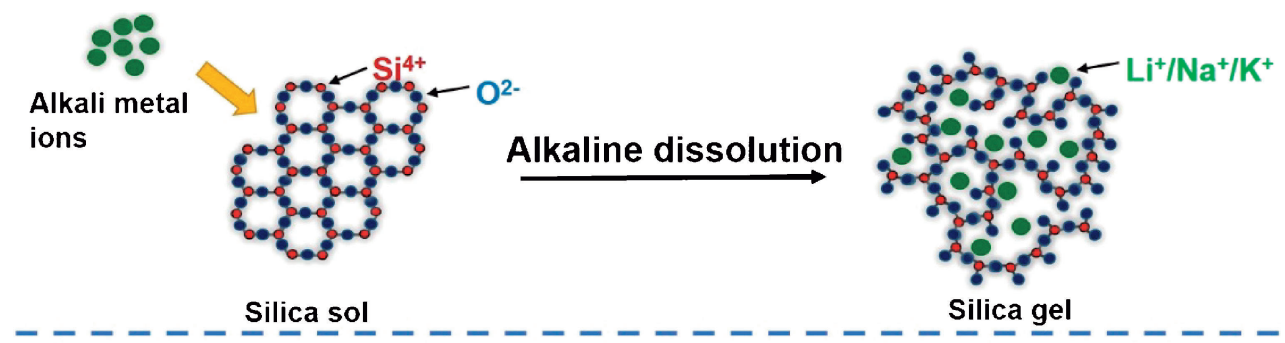

Microprocess demonstration (TEM)

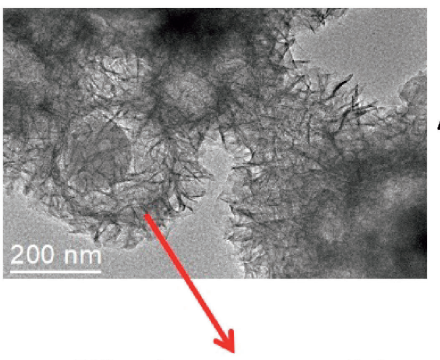

Silica fume nanoparticles

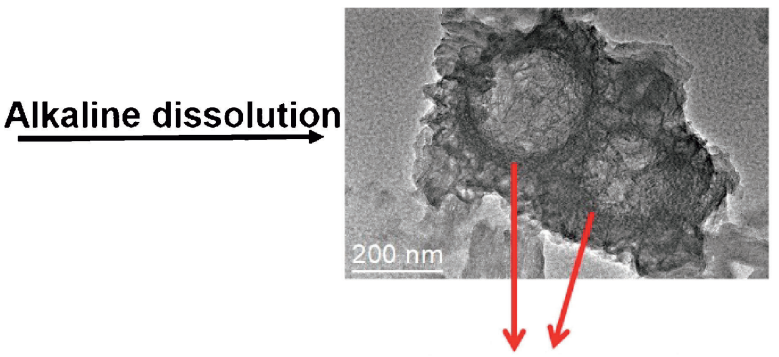

Silica fume nanoparticles

Fig. 8. Microscopic process of alkaline dissolution effect.

Table 3. Pore structure parameters of silica aerogels prepared with different alkaline catalysts

\begin{tabular}{cccccc}
\hline \multirow{2}{*}{ Samples } & $\begin{array}{c}\text { Specific surface } \\
\text { area }\end{array}$ & $\begin{array}{c}\text { Pore } \\
\text { volume }\end{array}$ & $\begin{array}{c}\text { Most probable } \\
\text { diameter }\end{array}$ & $\begin{array}{c}\text { Average pore } \\
\text { diameter }\end{array}$ \\
\cline { 2 - 6 } & $\mathrm{m}^{2} / \mathrm{g}$ & $\mathrm{cm}^{3} / \mathrm{g}$ & \multicolumn{2}{c}{$\mathrm{nm}$} & $\mathrm{nm}$ \\
\hline $\mathrm{T}-0$ & 772.6 & 4.139 & 1.688 & - & 21.43 \\
$\mathrm{~T}-1$ & 394.5 & 2.799 & 1.717 & 31.90 & 28.38 \\
$\mathrm{~T}-2$ & 427.0 & 3.349 & 1.704 & 32.17 & 31.37 \\
$\mathrm{~T}-3$ & 380.7 & 2.599 & 1.714 & 32.14 & 27.30 \\
$\mathrm{~T}-4$ & 413.9 & 3.238 & 1.545 & 32.39 & 31.29 \\
\hline
\end{tabular}

* T-0: None; T-1: LiOH; T-2: NaOH; T-3: $\mathrm{KOH}$; T-4: $\mathrm{NH}_{3} \cdot \mathrm{H}_{2} \mathrm{O}$.

Average pore diameter $=4 \times$ Pore volume/Specific surface area

alkaline catalysts were featured in a distinctive double-pore structure. There were two most probable diameters around 1.7 and $32 \mathrm{~nm}$ of silica aerogels T-1, T-2, T-3, and T-4. It was Indicated that the addition of alkaline catalysts would affect the formation of pores in the aerogels, promote the uniformity of pore-size, and generate a double-pore structure. It might be efficient in a barrier and mechanical property. Besides, the pore volume at $1.7 \mathrm{~nm}$ of silica aerogels T-1, T-2, and T-3 was significantly smaller than that at $32 \mathrm{~nm}$, while the two kinds of the pore volume of the silica aerogels T- 4 were similar. On the one hand, the former contained a large amount of alkali metal ions, which might produce the alkaline dissolution effect. On the other hand, the presence of alkali metal ions would block the hole at $1.7 \mathrm{~nm}$ but had little effect on the hole at $32 \mathrm{~nm}$.

The alkaline dissolution effect had been detected in the cement and silica fume system, which was called the Alkali-Silica Reaction in previous research. ${ }^{38)-40)}$ According to the above-mentioned characterizations, alkali metal ions in the silica aerogels system do have the alkaline dissolution effect during the sol-gel process. As displayed in Fig. 8, the initial states of the silica nanoparticles in sol is independent microspheres. In the weakly acid environ- ment, the silica nanoparticles in gel were getting even closer. So that the $\mathrm{Si}-\mathrm{O}-\mathrm{Si}$ groups were gradually forming with an open channel adsorbed with free water in the inner of silica gel, which would be exchanged by ethanol solution. Meanwhile, some of the alkali metal ions would enter the $\mathrm{Si}-\mathrm{O}-\mathrm{Si}$ groups. With the aging process proceeding, there were more and more alkali metal ions entering into the silica gel, accompanied by a stronger alkaline dissolution effect of alkali metal ions.

\section{Conclusion}

A series of experiments were conducted to investigate the influence of different alkaline catalysts on the formation of silica aerogels prepared by the sol-gel approach. The effects of different alkaline catalysts in terms of $\mathrm{LiOH}$, $\mathrm{NaOH}, \mathrm{KOH}$, and $\mathrm{NH}_{3} \cdot \mathrm{H}_{2} \mathrm{O}$ on the formation of silica aerogels were investigated. It was found that with the introduction of alkaline catalysts, the gel aging time dramatically decreased from 5840 to $31 \mathrm{~min}$ during the solgel process. Compared with the silica aerogels prepared without any catalyst, accordingly, the thermal stability was significantly improved, and the decomposition temperature increased from 376 to $400-410^{\circ} \mathrm{C}$. Also, the total mass loss of silica aerogels with alkaline catalysts containing alkali metal ions was lower than that with weak alkaline catalyst $\left(\mathrm{NH}_{3} \cdot \mathrm{H}_{2} \mathrm{O}\right)$. The alkaline dissolution effect had an influence on the pore size distribution of silica aerogels with a double pore structure at 1.7 and $32 \mathrm{~nm}$ as well as retained large pore volume at about $2.60-3.35 \mathrm{~cm}^{3} / \mathrm{g}$. This study could provide a better solution for the preparation and application of silica aerogels. Moreover, the detailed quantitative analysis is being explored in further study.

Acknowledgments The authors are grateful for the financial support from the National Natural Science Foundation of China (51303018). 
Conflict of interest We declare that we have no financial and personal relationships with other people or organizations that can inappropriately influence our work, there is no professional or other personal interest of any nature or kind in any product, service or company that could be construed as influencing the position presented in or the review of.

\section{References}

1) S. S. Kistler, Nature, 127, 741 (1931).

2) N. Hã Sing and U. Schubert, Angew. Chem. Int. Edit., 37, 22-45 (2010).

3) C. Zhao, Y. Yan, Z. Hu, L. Li and X. Fan, Constr. Build. Mater., 93, 309-316 (2015).

4) J. P. Randall, M. A. B. Meador and S. C. Jana, $A C S$ Appl. Mater. Interfaces, 3, 613-626 (2011).

5) J. E. Amonette and J. Matyáś, Micropor. Mesopor. Mat., 250, 100-119 (2017).

6) A. Veronovski, G. Tkalec, Ž. Knez and Z. Novak, Carbohyd. Polym., 113, 272-278 (2014).

7) T. W. Hamann, A. B. F. Martinson, J. W. Elam, M. J. Pellin and J. T. Hupp, Adv. Mater., 20, 1560-1564 (2008).

8) M. Schmidt, J. Non-Cryst. Solids, 225, 364-368 (1998).

9) J. Xu, X. Zhou, M. Chen, S. Shi and Y. Cao, Micropor. Mesopor. Mat., 265, 258-265 (2018).

10) D. Sun, K. Li, X. Sui, C. Zhou and F. Liu, J. Porous Mat., 25, 341-349 (2018).

11) A. Venkateswara Rao, S. D. Bhagat, H. Hirashima and G. M. Pajonkc, J. Colloid Interf. Sci., 300, 279-285 (2006).

12) Z. Li, X. Cheng, S. He, X. Shi, H. Yang and H. Zhang, Mater. Lett., 171, 91-94 (2016).

13) A. Venkateswara Rao, A. Parvathy Rao and M. M. Kulkarni, J. Non-Cryst. Solids, 350, 224-229 (2004).

14) C. J. Brinker, K. D. Keefer, D. W. Schaefer, R. A. Assink, B. D. Kay and C. S. Ashley, J. Non-Cryst. Solids, 63, 45-59 (1984).

15) S. Iswar, W. J. Malfait, S. Balog, F. Winnefeld, M Lattuada and M. M. Koebel, Micropor. Mesopor. Mat., 241, 293-302 (2017).

16) C. Lei, J. Li, C. Sun, H. Yang, T. Xia, Z. Hu and Y. Zhang, Micropor. Mesopor. Mat., 267, 107-114 (2018).

17) C. Lei, Z. Hu, Y. Zhang, H. Yang, J. Li, S. Hu, Micropor. Mesopor. Mat., 258, 236-243 (2018).

18) A. Venkateswara Rao and N. N. Parvathy, J. Mater. Sci., 28, 3021-3026 (1993).
19) S. Zhao, H. Xu, L. Wang and S. J. William, J. Wuhan Univ. Technol., 28, 476-482 (2013).

20) R. B. Torres, J. P. Vareda, A. Lamy-Mendes and L. Durães, J. Supercrit. Fluid., 147, 81-89 (2019).

21) P. B. Wagh, R. Begag, G. M. Pajonk, A. V. Rao and D. Haranath, Mater. Chem. Phys., 57, 214-218 (1999).

22) S. D. Bhagat, Y.-H. Kim, Y.-S. Ahn and J.-G. Yeo, Appl. Surf. Sci., 253, 3231-3236 (2007).

23) P. B. Sarawade, J.-K. Kim, A. Hilonga and H. T. Kim, Solid State Sci., 12, 911-918 (2010).

24) P. B. Sarawade, J.-K. Kim, A. Hilonga and H. T. Kim, Korean J. Chem. Eng., 27, 1301-1309 (2010).

25) P. B. Sarawade, J.-K. Kim, H.-K. Kim and H.-T. Kim, Appl. Surf. Sci., 254, 574-579 (2007).

26) M. A. Aegerter, N. Leventis and M. M. Koebel, "Aerogels Handbook" (2011) pp. 449-498.

27) F. He, J. Cheng, J.-Y. Wu, Y.-H. Huang, X.-H. Zhu and Z.-T. Qi, J. Sol-Gel Sci. Techn., 90, 323-329 (2019).

28) F. Shi, L. Wang, J. Liu and M. Zheng, J. Mater. Sci. Technol., 23, 402-406 (2007).

29) P. B. Sarawade, J.-K. Kim, A. Hilonga and H.-T. Kim, Powder Technol., 197, 288-294 (2010).

30) S. Lee, Y. C. Cha, H. J. Hwang, J.-W. Moon and I. S. Han, Mater. Lett., 61, 3130-3133 (2007).

31) J. Li, J. Cao, L. Huo and X. He, Mater. Lett., 87, 146149 (2012).

32) V. G. Parale, D. B. Mahadik, M. S. Kavale, A. V. Rao, P. B. Wagh and S. C. Gupta, Soft Nanosci. Lett., 1, 97104 (2011).

33) A. Hilonga, J.-K. Kim, P. B. Sarawade and H. T. Kim, J. Alloy. Compd., 487, 744-750 (2009).

34) M. V. Khedkar, S. B. Somvanshi, A. V. Humbe and K. M. Jadhav, J. Non-Cryst. Solids, 511, 140-146 (2019).

35) A. Y. Jeong, S. Koo and D. Kim, J. Sol-Gel Sci. Techn., 19, 483-487 (2000).

36) X. Tang, A. Sun, C. Chu, M. Yu, S. Ma, Y. Cheng, J. Guo and G. Xu, Mater. Design, 115, 415-421 (2017).

37) M. Thommes, K. Kaneko, A. V. Neimark, J. P. Olivier, F. Rodriguez-Reinoso, J. Rouquerol and K. S. W. Sing, Pure Appl. Chem., 87, 1051-1069 (2015).

38) S. Chatterji, N. Thaulow and A. D. Jensen, Cement Concrete Res., 18, 363-366 (1988).

39) H. Wang and J. E. Gillott, Cement Concrete Res., 21, 647-654 (1991).

40) E. Garcia-Diaz, J. Riche, D. Bulteel and C. Vernet, Cement Concrete Res., 36, 395-400 (2006). 